\title{
Construction and validation of a novel prognostic signature of microRNAs in lung adenocarcinoma
}

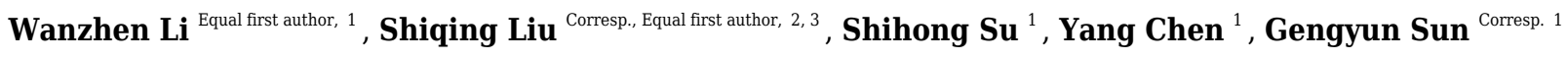 \\ ${ }^{1}$ Department of Respiratory and Critical Care Medicine, The First Affiliated Hospital of Anhui Medical University, Hefei, China \\ 2 Department of Respiratory and Critical Care Medicine, Xiangya Hospital, Central South University, Guangdong, China \\ ${ }^{3}$ Key cite of National Clinical Research Center for Respiratory Disease, Xiangya Hospital, Central South University, Guangdong, China \\ Corresponding Authors: Shiqing Liu, Gengyun Sun \\ Email address: 451063788@qq.com, sungengy@126.com
}

MicroRNA (miRNA, miR) has been reported to be highly implicated in a wide range of biological processes in lung cancer (LC), and identification of differentially expressed miRNAs between normal and LC samples has been widely used in the discovery of prognostic factors for overall survival (OS) and response to therapy. The present study was designed to develop and evaluate a miRNA-based signature with prognostic value for the OS of lung adenocarcinoma (LUAD), a common histologic subtype of LC. In brief, the miRNA expression profiles and clinicopathological factors of 499 LUAD patients were collected from The Cancer Genome Atlas (TCGA) database. Kaplan-Meier (K-M) survival analysis showed significant correlations between differentially expressed miRNAs and LUAD survival outcomes. Afterward, 1,000 resample LUAD training matrices based on the training set was applied to identify the potential prognostic miRNAs. The least absolute shrinkage and selection operator (LASSO) cox regression analysis was used to constructed a six-miRNA based prognostic signature for LUAD patients. Samples with different risk scores displayed distinct OS in K-M analysis, indicating considerable predictive accuracy of this signature in both training and validation sets. Furthermore, time-dependent receiver operating characteristic (ROC) analysis demonstrated the nomogram achieved higher predictive accuracy than any other clinical variables after incorporating the clinical information (age, sex, stage, and recurrence). In the stratification analysis, the prognostic value of this classifier in LUAD patients was validated to be independent of other clinicopathological variables, such as age, gender, tumor recurrence, and early stage. Gene set annotation analyses were also conducted through Hallmark gene set and Kyoto Encyclopedia of Genes and Genomes (KEGG) pathways, indicating target genes of the six miRNAs were positively related to various molecular pathways of cancer, such as hallmark UV response, Wnt signaling pathway and mTOR signaling pathway. In addition, fresh cancer tissue samples and matched adjacent tissue samples from 12 LUAD patients were 
collected to verify the expression of miR-582's target genes in the model, further revealing the potential relationship between SOX9, RASA1, CEP55, MAP4K4 and LUAD tumorigenesis, and validating the predictive value of the model. Taken together, the present study identified a robust signature for the OS prediction of LUAD patients, which could potentially aid in the individualized selection of therapeutic approaches for LUAD patients. 
1 Construction and Validation of a Novel Prognostic Signature of MicroRNAs in Lung

2 Adenocarcinoma

3

1Department of Respiratory and Critical Care Medicine, the First Affiliated Hospital of Anhui Medical University, Hefei,

6 China.

$7 \quad{ }^{2}$ Department of Respiratory and Critical Care Medicine, Xiangya Hospital, Central South University

${ }^{3}$ Key cite of National Clinical Research Center for Respiratory Disease, Xiangya Hospital, Central South University

\#These authors contributed equally to the work.

Correspondence to: *Corresponding to Shiqing Liu (451063788@qq.com) \& Gengyun Sun (sungengy@126.com)

\section{Abstract}

MicroRNA (miRNA, miR) has been reported to be highly implicated in a wide range of biological processes in lung cancer (LC), and identification of differentially expressed miRNAs between normal and LC samples has been widely used in the discovery of prognostic factors for overall survival (OS) and response to therapy. The present study was designed to develop and evaluate a miRNA-based signature with prognostic value for the OS of lung adenocarcinoma (LUAD), a common histologic 
subtype of LC. In brief, the miRNA expression profiles and clinicopathological factors of 499 LUAD patients were collected from The Cancer Genome Atlas (TCGA) database. Kaplan-Meier (K-M) survival analysis showed significant correlations between differentially expressed miRNAs and LUAD survival outcomes. Afterward, 1,000 resample LUAD training matrices based on the training set was applied to identify the potential prognostic miRNAs. The least absolute shrinkage and selection operator (LASSO) cox regression analysis was used to constructed a six-miRNA based prognostic signature for LUAD patients. Samples with different risk scores displayed distinct OS in K-M analysis, indicating considerable predictive accuracy of this signature in both training and validation sets. Furthermore, time-dependent receiver operating characteristic (ROC) analysis demonstrated the nomogram achieved higher predictive accuracy than any other clinical variables after incorporating the clinical information (age, sex, stage, and recurrence). In the stratification analysis, the prognostic value of this classifier in LUAD patients was validated to be independent of other clinicopathological variables, such as age, gender, tumor recurrence, and early stage. Gene set annotation analyses were also conducted through Hallmark gene set and Kyoto Encyclopedia of Genes and Genomes (KEGG) pathways, indicating target genes of the six miRNAs were positively related to various molecular pathways of cancer, such as hallmark UV response, Wnt signaling pathway and mTOR signaling pathway. In addition, fresh cancer tissue samples and matched adjacent tissue samples from 12 LUAD patients were collected to verify the expression of miR-582's target genes in the model, further revealing the potential relationship between SOX9, RASA1, CEP55, MAP4K4 and LUAD tumorigenesis, and validating the predictive value of the model. Taken together, the present study identified a robust signature for the OS prediction of LUAD patients, which could potentially aid in the 
individualized selection of therapeutic approaches for LUAD patients.

41 Keywords: LASSO bagging cox regression test, lung adenocarcinoma, TCGA, prognosis

\section{Introduction}

Lung cancer (LC) is the leading cause of malignancy-related mortality, with approximately one million cases of death annually, which is a great burden for public health worldwide (Torre et al., 2015). Lung adenocarcinoma (LUAD) is the most common histologic subtype of LC (Brambilla et al., 2001; Wakelee et al., 2007), accounting for $50 \%$ of LC cases. Despite the remarkable progress in image scan, bronchoscopy, and novel therapeutic options (Sun et al., 2007; Minna et al., 2002), tumor progression remains the major obstacle for a favorable survival rate. Additionally, $40 \%$ of stage

IB and $66 \%$ of stage II patients are facing a high recurrence rate within 5 years, which is associated with a poor prognosis (Chansky et al., 2010). Recent studies have shown that adjuvant chemotherapy confers a $4-15 \%$ survival benefit for stage II-III patients receiving tumor resection rather than patients with stage I (Reck et al., 2013; Pignon et al., 2008). The limited survival benefits suggest the insufficient power of the currently available staging system and the presence of more undiscovered tumor heterogeneity. Therefore, it is imperative to identify early diagnostic markers and establish effective predictive systems for prognosis to improve the survival of LUAD patients. 
expression at a post-transcriptional level (Garzon et al., 2009). By specifically combing to the 3'untranslated region (UTR) of target genes and affecting messenger RNA (mRNA), miRNA has been proved to be involved in the regulation of basic cellular processes, including cell differentiation, proliferation, and apoptosis (Winter et al., 2009). Differentially expression levels of miRNAs, which may exhibit either oncogenic or tumor-suppressive activity, are reported to play crucial roles in the prognosis of LC (Li et al., 2019a; Li et al., 2019b; Liu et al., 2018; Garzon et al., 2009; Lujambio et al., 2007). The role of miRNA in lung carcinogenesis was first proposed in 2004 by Calin et al. (Calin et al., 2004), who demonstrated that half of the miRNA genes were located at cancer-related genomic regions or in fragile sites, and several miRNAs in these regions had low expression level in LC cell lines. Consistently, the down-regulation of the let-7 miRNA was found by another study published in the same year (Takamizawa et al., 2004), and acted as onco-suppressor role in LUAD carcinogenesis (Kumar et al., 2008). Junichi et al. have shown that down-regulation of the let-7 miRNA could prohibit the expression of RAS, MYC, and HMGA2 oncogenes (Lee \& Dutta, 2007; Kumar et al., 2007). While Johnson et al. have reported that the over-expression of let-7 in a LC cell line (A549) could cause cell cycle arrest and inhibit cell growth (Johnson et al., 2005). The miR-34 family, consisting of miR-34a, $\mathrm{miR}-34 \mathrm{~b}$, and miR-34c members, has long been reported to be involved in the p53 regulatory network (He et al., 2007). By directly targeting MET and BCL2, the downregulation of miR-34 could promote cell growth and proliferation (Bommer et al., 2007). Besides, a novel tumor evasion mechanism has been proposed, indicating that PDL1 was regulated by p53 via miR-34 in PD1/PDL1 signaling in nonsmall cell lung cancer (NSCLC) patients (Cortez et al., 2016). 
Currently, miRNAs have been increasingly used not only as diagnostic but also prognostic biomarkers due to the convenient detection in body fluids such as blood, serum, urine, and sputum.

Several studies have suggested the existence of tissue-specific miRNA signatures, which might be used to classify di $\square$ erent cancer types (Zhang et al., 2019; Xiong et al., 2018; DeSantis et al., 2014; Chansky et al., 2010). Therefore, it is promising to find out some prognosis-related miRNA markers to establish the prognostic prediction signature. Herein, we established an overall survival (OS) predictive signature based on a six-miRNA-based panel, followed by validation of its usage and stability in prognostic prediction in LUAD patients.

\section{Material and Methods}

Data and sources with the clinical information of related samples including survival follow-up data, age, gender, tumor node metastasis (TNM) stage, and tumor recurrence. The miRNA expression data from the LUAD samples were based on IlluminaHiSeq_miRNASeq, followed by quantile normalization and log2scale transformation by $\mathrm{R}$ software. All the enrolled samples were 2:1 random sampling without replacement by using sample $\mathrm{R}$ package. The randomizing process was blind to the demographic information or clinical information, which could ensure that the training and validation cohorts are 
97

98

99

100

101

102

104

105

106

107

108

109

110

111

112

113

114

115

independent to clarify the key points we focused on. We use R (http://www.R-project.org) software to make all statistical analyses. A two-sided $\mathrm{P}<0.05$ was considered statistically significant.

Herein, the predictive model was initially constructed in the training set, and the validation group was employed to provide the unbiased evaluation of the final model in the training set.

In addition,12 cases of fresh LUAD tissue samples and paired adjacent cancer tissue samples were enrolled from the Department of Thoracic Surgery, the First Affiliated Hospital of Anhui Medical University.

All the 24 surgically resected samples were immersed in $10 \%$ formalin. Informed consents were signed by all patients and our study was approved by the Ethics Committee of the First Affiliated Hospital of Anhui Medical University (anyiyifuyuanlunshen-Quik-PJ-2019-09-09) (anyiyifuyuanlunshen-Quik-PJ-2020-05-08). For the patients collected from the TCGA project, the ethics and policies were announced on their Website (https://www.cancer.gov/aboutnci/organization/ccg/research/structural-genomics/tcga/history/policies).

Establishment and validation of a miRNA signature with the least absolute shrinkage and selection operator (LASSO) regression model (K-M) survival analysis, followed by a log-rank test to select all the potential miRNAs. The candidate miRNA was considered to be significantly associated with the OS of LUAD patients when the $P$-value was less than 0.05 (Xu et al., 2017). A group of 1,000 training matrices based on the LUAD patients 
116

117 (RMIP). Followed, with the R package "glmnet" (2.0-10), the LASSO bagging cox regression test, the classical and modified method in Cox regression analysis of high dimensional data was performed with 10 -fold cross-validation on all the OS related matrices (Tibshirani, 1997). The tuning parameter $\lambda$ was determined by the standard error (1-SE), which introduced the penalties to the model construction process to avoid over-fitting. The larger $\lambda$ was, the more the coefficients were shrunk toward zero (Goeman, 2010). In this study, the recommended $\lambda$ value with minimal mean squared error plus one standard deviation was selected in each Lasso cox regression test. A group of 1,000

formula. Afterward, the LUAD samples with assigned risk scores were classified into a high-risk or

low-risk group by using the median as the cutoff point. To confirm the predictive ability of this model, 
136

137

138

139

140

141

142

143

144

145

146

147

distinguish the survival difference between the high-risk group and low-risk group. A two-tailed $P$ value $<0.05$ in $\mathrm{K}-\mathrm{M}$ analyses was considered as statistically significant. In addition, to graphically show the diagnostic value of the classifier system, receiver operating characteristic (ROC) analysis was adopted to compare the sensitivity and specificity of survival prediction. Youden's index $=\max$ [1- (sensitivity + specificity)] (Hilden \& Glasziou, 1996). Moreover, the area under the ROC curve (AUC) for evaluating discriminatory ability was calculated as well.

\section{Network and annotation analysis of target genes}

We obtained the target genes from TargetScan, miRTarBase, and miRDB (Wong \& Wang, 2015; Chou et al., 2018; Agarwal et al., 2015), respectively. To obtain more convincing results, we overlapped the target genes from the three websites and generated a new target gene cluster. Cytoscape software (v3.5.1, San Diego, La Jolla, California 92093, USA) was used for the visualization of the interactive network to assist the understanding miRNA function and regulatory mechanisms. Besides, Hallmark gene set and Kyoto Encyclopedia of Genes and Genomes (KEGG) analyses were performed to further study the functional roles of the target genes during LUAD progression using the R package "clusterProfiler" (Q-value < 0.05) (Yu et al., 2012).

\section{Immunohistochemistry (IHC)}


155 IHC validation, which was performed according to the previous description (Zhang et al., 2019). The t-

156 test was used to determine the protein expression differences between LUAD tissue specimens and

157 paired adjacent cancer tissue specimens. A two-tailed $P$-value $<0.05$ was considered as statistically

158 significant. The SOX9, RASA1, CEP55 and MAP4K4 antibodies (catalog \# GR3253329-3; GR85944-

159 25; GR234851-12; 09000421) were purchased from Abcam (Cambridge, U.K.,

160 http://www.abcam.com) and Proteintech (Chicago, IL, http://www.ptglab.com).

162 Results

Construction of a prognostic signature based on miRNAs

from the TCGA databases. OS information was available in 499 cases, which were further retained as the training set (329 samples) and validation set (170 samples).

The K-M survival analysis was employed for the preliminary screening of potential OS related miRNAs (Figure S1). As a result, we selected eight miRNAs based on $P<0.05$, which indicated significant correlation with the LUAD patients' OS. Afterwards, we rebuilt 1,000 resample LUAD training matrices based on the training cohort patients. The RMIP of each OS-related miRNA was listed and ordered from the most frequent one to the least miRNA (Figure 1A). According to the LASSO cox regression analysis on performance of all the OS related matrices with 10 -fold cross validation, six 
174

175

176

177

178

179

180

181

182

183

miRNAs were acquired, including miR-1468 $(\mathrm{HR}=0.69,95 \% \mathrm{Cl}=0.560-0.870, P=0.001$, co-ef $=-$ 0.366), miR-299 $(\mathrm{HR}=1.48,95 \% \mathrm{Cl}=1.220-1.800, P<0.001$, co-ef $=0.394)$, miR-4709 $(\mathrm{HR}=0.720$, $95 \% \mathrm{Cl}=0.590-0.890, P=0.002$, co-ef $=-0.322)$, miR-5571 $(\mathrm{HR}=0.570,95 \% \mathrm{Cl}=0.370-0.860, P=$ 0.008 , co-ef $=-0.570)$, miR-582 $(\mathrm{HR}=1.18,95 \% \mathrm{Cl}=1.050-1.320, P=0.005$, co-ef $=0.166)$, and $\operatorname{miR}-3653(\mathrm{HR}=0.87,95 \% \mathrm{Cl}=0.730-1.030, P=0.106$, co-ef $=-0.143)($ Figure 1B $)$. A risk score model for each patient was constructed to predict prognosis of LUAD patients by including the expression level of each optimal prognostic miRNA, weighted by their estimated regression coefficients of LASSO Bagging Cox regression model as $=-0.365{ }^{*} \mathrm{miR}-1468+0.394{ }^{*} \mathrm{miR}-299-$ 0.322 * miR-4709 -0.570 * miR-5571 + 0.166 * miR-582 - 0.143 * miR-3653.

\section{Validation and evaluation of the miRNAs signature}

or low-risk group. The K-M curve with the log-rank test demonstrated significantly different survival between high-risk and low-risk groups in both training $(P<0.0001$, Figure 2B) and validation groups $(P=0.0085$, Figure 2D). To evaluate the classification potential of this model, the ROC analysis was performed and AUC was calculated in both training and validation cohorts. As shown in Figure 2A and Figure 2C, the AUC of ROC was 0.72 and 0.70 in the training group and validation group, respectively. Besides, considering other partitions to training and validation groups can both increase and decrease the reliability of the classifier, we repeated the step of different training and validation groups multiple times and efficiently calculated the means/variations/confidence intervals for the 
194

195

196

197

198

199

sensitivity, specificity and AUC to validate the reliability of the classifier as the Supplementary Table 3.

These results proved that the classifier has moderate discriminatory power and potential utility in determining the LUAD patients with low or high risk as presenting high and stable of AUC and sensitivity.

Moreover, nomograms were constructed in the training, validation, and overall groups after incorporating the clinical characteristics (age, sex, TNM stage, and tumor recurrence) (Figure 3A-3C). The results of ROC analyses identified this six-miRNAs classifier as the most accurate contributor to prognosis compared to other clinical factors, and the nomogram could precisely and steadily judge the OS rate of LUAD.

\section{Subgroup analysis}

Age, TNM stage, and tumor recurrence are always important clinical factors influencing the prognosis of LUAD. Therefore, stratification analyses based on TNM stage, tumor recurrence, and other clinical variables were performed to validate whether this classifier had functional discriminatory capacity among different clinical status (Figure 4). According to the TNM staging system, LUAD patients have categorized two groups (stage I/II, stage III/IV). Within Stage I/II, the OS related to the six miRNAs signature prognostic value remains significantly different (stage $\mathrm{I} / \mathrm{Il}: P<0.001$ ), but not in Stage III/IV subgroup (stage III/IV: $P=0.061$ ). When adjusted by age, sex, and new tumor event after initial treatment, our risk score model still exhibited the reliability and general applicability for 
213 distinguishing each group among different clinical factors.

214

Functional annotation analysis and IHC Validation

216

The target genes of miR-1468, miR-299, miR-4709, miR-5571, and miR-582 were predicted by the online prediction tool like TargetScan, miRTarBase, and miRDB. The miRNA-miRNA regulatory network was shown in Figure 5. Additionally, we performed functional annotation analysis for Hallmark gene set and KEGG pathways (Figure 6), demonstrating that target genes of different miRNAs were significantly enriched in several functional pathways related to cancer development, such as hallmark UV response, Wnt signaling pathway and mTOR signaling pathway. expression of SOX9, RASA1, CEP55, and MAP4K4 was detected in LUAD clinical specimens by IHC. protein expression $(p<0.05)$ (Figure S2-S5).

\section{Discussion}

Characterized by the tendency of an advanced stage and metastatic tumor, LUAD confronts 
231 outcomes, though it accounts for only 25\%-30\% of LC (DeSantis et al., 2014; Scott et al., 2007).

232 Surgical resection with or without additional adjuvant chemotherapy is regarded as the cornerstone of

233 therapeutic treatment for early-stage LUAD, and TNM stage is the most significant biomarker to

234 predict prognosis traditionally (Crinò et al., 2010; Vansteenkiste et al., 2014; Cheng et al., 2012).

235 Whereas, survival varies greatly, even within the same-staged LUAD receiving the same treatment,

236 demonstrating the genetic heterogeneity among LUAD.

In recent years, substantial cancer-related studies have focused on miRNAs profiles, as

exceptionally influencing developmental and oncogenic pathways by regulating gene expression.

Aberrant expression of miRNAs is considered as hallmark features in the biological progression of

various diseases, including LC (Ortholan et al., 2009; Saito et al., 2011; Patnaik et al., 2010).

Integration of miRNAs and target genes profiles is a promising approach to identify effective

biomarkers of LUAD (Zhu et al., 2019; Zhao et al., 2013; Jacob et al., 2018). In our work, we obtained

the miRNA expression profiles from TCGA databases and subsequently constructed a six-miRNAs

based predictive signature of the prognosis for LUAD patients. This miRNAs-based signature could classify the high-risk group and low-risk group of LUAD with distinct difference survival. After incorporating the clinical parameters (age, sex, TNM stage, and tumor recurrence), the six-miRNA based nomogram exhibited favorable discriminative performance. In respect to stratification analyses based on clinicopathological variables, we validated the potential clinical utility of miRNA-based predictive signature for LUAD's OS, independent of age ( $>60$, and $<60)$, gender, tumor recurrence, and the early stage. 
251

252

253

254

255

256

257

258

259

260

261

262

263

264

265

266

267

268

269

In consideration of the individual roles of six miRNAs (miR-1468, miR-299, miR-4709, miR-5571, miR-582, and miR-3653), several studies have reported their imperative involvements in prognosis value of LC as well. Niu et al. investigated that the mRNA expression of PIP4K2A regulated by hsamiR-1468 was directly correlated with the OS of paclitaxel-treated LC (Niu et al., 2012). When the differentially expressed miRNAs were subjected to univariate Cox proportional hazard regression analysis, Lin et al. pointed out that miR-1468 $(P=0.009)$ and miR-3653 $(P=0.012)$ were significantly correlated with recurrence-free survival (RFS) of LUAD (Lin et al., 2016). The down-regulated expression of miR-299 in LUAD has been reported to serve as a functional role in therapeutic efficacy, including gefitinib resistance and chemoresistance (Negrete-Garcia et al., 2018; Li et al., 2018; Zheng et al., 2015). Through miR-299-3p/TNFSF12 pathway, RHPN1-AS1 could modulate the LUAD therapeutic resistance to gefitinib (Li et al., 2018), and the suppression of ABCE1 could affect the sensitivity of LC to doxorubicin (Zheng et al., 2015). Based on the detection in both tissues and plasma of LUAD patients with different stages, $\mathrm{Pu}$ et al. revealed that constantly expressed miR5571-5p was a promising biomarker of LUAD with different stages (Pu et al., 2016), which was consistent with the study by Zheng et al. (Zheng et al., 2018). MiR-4709 and miR-582 in this signature were initially documented as prognostic markers in different tumors (Siriwardhana et al., 2019; Xin et al., 2019), which were validated by the microarray technology or TCGA. The miR-582 was further shown to be significantly down-regulated in NSLCLC cell lines, which could suppress the proliferation, migration, and invasion of NSLCLC cells by targeting MAP3K2 (Wang \& Zhang, 2018). 
We also presented the biological functions of these six miRNAs at the molecular level using the

271 Hallmark gene set and KEGG analyses. As a result, the identified targets genes were mainly enriched

272 in cancer-related pathways, such as hallmark UV response, Wnt signaling pathway, and mTOR

273 signaling pathway. Wnt signaling pathways could significantly affect tumorigenesis, prognosis, and

274 therapeutic resistance in NSCLC (Stewart, 2014). Activation of FOXP3 could facilitate the Wnt- $\beta$ -

catenin signaling pathway in epithelial-mesenchymal transition (EMT), tumor growth, and metastasis

of LUAD (Yang et al., 2017). MTOR signaling pathways were involved in regulating autophagy, apoptosis, proliferation, and metastasis in the pathophysiology of LUAD (Liu et al., 2017; Han et al.,

2013; Yu et al., 2017). In order to assess the potential target genes of miRNAs, IHC was used to

randomly identify the expression of SOX9, RASA1, CEP55 and MAP4K4, the target genes of miR-

582. The results revealed that SOX9, RASA1, CEP55, and MAP4K4 were significantly higher

expressed in LUAD tissues than those in adjacent cancer tissues. As wang et al. have validated that

the expression of miR-582 was significantly down-regulated in NSLCLC cell lines and clinical

specimens (Wang \& Zhang, 2018), it is hypothesized that down-regulated miR-582 could inversely

lead to the over-expression of its target genes, which is consistent with our results. However, the

biological function of these target genes in LUAD has not been investigated in our study. In further

research, we will conduct well-designed and comprehensive functional and mechanistic experiments.

In summary, we have identified six miRNAs related to the OS of LUAD and constructed a 
290

291

292

293

294

295

296

297

298

299

300

301

302

303

304

305

306

treatment decisions for LUAD patients. By randomly assessing the potential target genes in the tumor samples, we have found that up-regulated SOX9, RASA1, CEP55 and MAP4K4, the target genes of miR-582, might play important roles in LUAD tumorigenesis. However, the underlying mechanism should be further investigated. Collectively, our model is promising for clinical prognostic evaluation of LUAD patients.

\section{Acknowledgments}

None

\section{Funding Sources}

This work was supported by the National Key Clinical Specialist Construction Programs of China (N3101005005025).

\section{Conflicts of interests}

None.

\section{Author Contributions}


collection, or analysis and interpretation of data; W.L. and S.L. drafting the article or revising it

critically; G.S. final approval of the version to be submitted.

310

\section{Reference:}

Agarwal V, Bell GW, Nam JW, and Bartel DP. 2015. Predicting effective microRNA target sites in mammalian mRNAs. Elife 4. 10.7554/eLife.05005

Bommer GT, Gerin I, Feng Y, Kaczorowski AJ, Kuick R, Love RE, Zhai Y, Giordano TJ, Qin ZS, Moore BB, MacDougald OA, Cho KR, and Fearon ER. 2007. p53-mediated activation of miRNA34 candidate tumor-suppressor genes. Curr Biol 17(15):1298-1307. 10.1016/j.cub.2007.06.068

Brambilla E, Travis WD, Colby TV, Corrin B, and Shimosato Y. 2001. The new World Health Organization classification of lung tumours. Eur Respir J 18(6):1059-1068. 10.1183/09031936.01.00275301

Calin GA, Sevignani C, Dumitru CD, Hyslop T, Noch E, Yendamuri S, Shimizu M, Rattan S, Bullrich F, Negrini M, and Croce CM. 2004. Human microRNA genes are frequently located at fragile sites and genomic regions involved in cancers. Proc Natl Acad Sci U S A 101(9):2999-3004. 10.1073/pnas.0307323101

Chansky K, Sculier JP, Crowley JJ, Giroux D, Van Meerbeeck J, and Goldstraw P. 2010. [The International Association for the Study of Lung Cancer Staging Project. Prognostic factors and pathologic TNM stage in surgically managed non-small cell lung cancer]. Zhongguo Fei Ai Za Zhi 13(1):9-18. 10.3779/j.issn.1009-3419.2010.01.02

Cheng YD, Duan CJ, Dong S, Zhang H, Zhang SK, Wang SQ, and Zhang CF. 2012. Clinical controlled comparison between lobectomy and segmental resection for patients over 70 years of age with clinical stage I non-small cell lung cancer. Eur $J$ Surg Oncol 38:1149-1155. 10.1016/j.ejso.2012.08.001

Chou CH, Shrestha S, Yang CD, Chang NW, Lin YL, Liao KW, Huang WC, Sun TH, Tu SJ, Lee WH, Chiew MY, Tai CS, Wei TY, Tsai TR, Huang HT, Wang CY, Wu HY, Ho SY, Chen PR, Chuang CH, Hsieh PJ, Wu YS, Chen WL, Li MJ, Wu YC, Huang XY, Ng FL, Buddhakosai W, Huang PC, Lan KC, Huang CY, Weng SL, Cheng YN, Liang C, Hsu WL, and Huang HD. 2018. miRTarBase update 2018: a resource for experimentally validated microRNA-target interactions. Nucleic Acids 
335

336

337

338

339

340

341

342

343

344

345

346

347

348

349

350

351

352

353

354

355

356

Res 46(D1):D296-D302. 10.1093/nar/gkx1067

Cortez MA, Ivan C, Valdecanas D, Wang X, Peltier HJ, Ye Y, Araujo L, Carbone DP, Shilo K, Giri DK, Kelnar K, Martin D, Komaki R, Gomez DR, Krishnan S, Calin GA, Bader AG, and Welsh JW. 2016. PDL1 Regulation by p53 via miR-34. J Natl Cancer Inst 108(1): djv303. 10.1093/jnci/djv303

Crinò L, Weder W, van Meerbeeck J, and Felip E. 2010. Early stage and locally advanced (non-metastatic) non-small-cell lung cancer: ESMO Clinical Practice Guidelines for diagnosis, treatment and followup. Ann Oncol 21 Suppl 5:v103-115. 10.1093/annonc/mdq207

DeSantis CE, Lin CC, Mariotto AB, Siegel RL, Stein KD, Kramer JL, Alteri R, Robbins AS, and Jemal A. 2014. Cancer treatment and survivorship statistics, 2014. CA Cancer J Clin 64(4):252-271. $10.3322 /$ caac. 21235

Garzon R, Calin GA, and Croce CM. 2009. MicroRNAs in Cancer. Annu Rev Med 60:167-179. 10.1146/annurev.med.59.053006.104707

Goeman JJ. 2010. L1 penalized estimation in the Cox proportional hazards model. Biom J 52(1):70-84. 10.1002/bimj.200900028

Han D, Li SJ, Zhu YT, Liu L, and Li MX. 2013. LKB1/AMPK/mTOR signaling pathway in non-small-cell lung cancer. Asian Pac J Cancer Prev 14(7):4033-4039. 10.7314/apjcp.2013.14.7.4033

He L, He X, Lim LP, de Stanchina E, Xuan Z, Liang Y, Xue W, Zender L, Magnus J, Ridzon D, Jackson AL, Linsley PS, Chen C, Lowe SW, Cleary MA, and Hannon GJ. 2007. A microRNA component of the p53 tumour suppressor network. Nature 447(7148):1130-1134. 10.1038/nature05939

Hilden J, and Glasziou P. 1996. Regret graphs, diagnostic uncertainty and Youden's Index. Stat Med15(10):969-986.10.1002/(sici)1097-0258(19960530)15:10<969::aidsim211>3.0.co;2-9

Jacob H, Stanisavljevic L, Storli KE, Hestetun KE, Dahl O, and Myklebust MP. 2018. A four-microRNA classifier as a novel prognostic marker for tumor recurrence in stage II colon cancer. Sci Rep 8(1):6157. 10.1038/s41598-018-24519-4

Johnson SM, Grosshans H, Shingara J, Byrom M, Jarvis R, Cheng A, Labourier E, Reinert KL, Brown D, and Slack FJ. 2005. RAS is regulated by the let-7 microRNA family. Cell 120(5):635-647. 10.1016/j.cell.2005.01.014

Kumar MS, Erkeland SJ, Pester RE, Chen CY, Ebert MS, Sharp PA, and Jacks T. 2008. Suppression of non-small cell lung tumor development by the let-7 microRNA family. Proc Natl Acad Sci U S A 105(10):3903-3908. 10.1073/pnas.0712321105 
Kumar MS, Lu J, Mercer KL, Golub TR, and Jacks T. 2007. Impaired microRNA processing enhances cellular transformation and tumorigenesis. Nat Genet 39(5):673-677. 10.1038/ng2003

Lee YS, and Dutta A. 2007. The tumor suppressor microRNA let-7 represses the HMGA2 oncogene. Genes Dev 21(9):1025-1030. 10.1101/gad.1540407

Li H, Liu J, Cao W, Xiao X, Liang L, Liu-Smith F, Wang W, Liu H, Zhou P, Ouyang R, Yuan Z, Liu J, Ye M, and Zhang B. 2019a. C-myc/miR-150/EPG5 axis mediated dysfunction of autophagy promotes development of non-small cell lung cancer. Theranostics 9:5134-5148. 10.7150/thno.34887

Li X, Zhang X, Yang C, Cui S, Shen Q, and Xu S. 2018. The IncRNA RHPN1-AS1 downregulation promotes gefitinib resistance by targeting miR-299-3p/TNFSF12 pathway in NSCLC. Cell Cycle 17(14):1772-1783. 10.1080/15384101.2018.1496745

Li X, Zhu J, Liu Y, and Duan C. 2019b. MicroRNA-331-3p inhibits epithelial-mesenchymal transition by targeting ErbB2 and VAV2 through the Rac1/PAK1/ $\beta$-catenin axis in non-small-cell lung cancer. Cancer Sci. 110:1883-1896. 10.1111/cas.14014

Lin K, Xu T, He BS, Pan YQ, Sun HL, Peng HX, Hu XX, and Wang SK. 2016. MicroRNA expression profiles predict progression and clinical outcome in lung adenocarcinoma. Onco Targets Ther 9:5679-5692. 10.2147/ott.s111241

Liu N, Liu Z, Zhang W, Li Y, Cao J, Yang H, and Li X. 2018. MicroRNA-433 reduces cell proliferation and invasion in non-small cell lung cancer via directly targeting E2F transcription factor 3. Mol Med Rep 18:1155-1164. 10.3892/mmr.2018.9020

Liu G, Pei F, Yang F, Li L, Amin AD, Liu S, Buchan JR, and Cho WC. 2017. Role of Autophagy and Apoptosis in Non-Small-Cell Lung Cancer. Int J Mol Sci 18(2):367. 10.3390/ijms18020367

Lujambio A, Ropero S, Ballestar E, Fraga MF, Cerrato C, Setién F, Casado S, Suarez-Gauthier A, Sanchez-Cespedes M, Git A, Spiteri I, Das PP, Caldas C, Miska E, and Esteller M. 2007. Genetic unmasking of an epigenetically silenced microRNA in human cancer cells. Cancer Res 67(7):1424-1429. 10.1158/0008-5472.can-06-4218

Minna JD, Roth JA, and Gazdar AF. 2002. Focus on lung cancer. Cancer cell 1:49-52. 10.1016/s15356108(02)00027-2

Negrete-Garcia MC, Ramírez-Rodriguez SL, Rangel-Escareño C, Muñoz-Montero S, Kelly-García J, Vázquez-Manríquez ME, Santillán P, Ramírez MM, Ramírez-Martínez G, Ramírez-Venegas A, and Ortiz-Quintero B. 2018. Deregulated MicroRNAs in Cancer-Associated Fibroblasts from Front 
395

396

397

398

399

400

401

402

403

404

405

406

407

408

409

410

411

412

413

414

415

416

417

418

419

420

421

422

423

424

Tumor Tissues of Lung Adenocarcinoma as Potential Predictors of Tumor Promotion. Tohoku J Exp Med 246(2):107-120. 10.1620/tjem.246.107

Niu N, Schaid DJ, Abo RP, Kalari K, Fridley BL, Feng Q, Jenkins G, Batzler A, Brisbin AG, Cunningham JM, Li L, Sun Z, Yang P, and Wang L. 2012. Genetic association with overall survival of taxanetreated lung cancer patients - a genome-wide association study in human lymphoblastoid cell lines followed by a clinical association study. Bmc Cancer 12:422. 10.1186/1471-2407-12-422

Ortholan C, Puissegur MP, Ilie M, Barbry P, Mari B, and Hofman P. 2009. MicroRNAs and lung cancer: new oncogenes and tumor suppressors, new prognostic factors and potential therapeutic targets. Curr Med Chem 16(9):1047-1061. 10.2174/092986709787581833

Patnaik SK, Kannisto E, Knudsen S, and Yendamuri S. 2010. Evaluation of microRNA expression profiles that may predict recurrence of localized stage I non-small cell lung cancer after surgical resection. Cancer Res 70(1):36-45. 10.1158/0008-5472.can-09-3153

Pignon JP, Tribodet H, Scagliotti GV, Douillard JY, Shepherd FA, Stephens RJ, Dunant A, Torri V, Rosell R, Seymour L, Spiro SG, Rolland E, Fossati R, Aubert D, Ding K, Waller D, and Le Chevalier T. 2008. Lung adjuvant cisplatin evaluation: a pooled analysis by the LACE Collaborative Group. $J$ Clin Oncol 26(21):3552-3559. 10.1200/jco.2007.13.9030

Pu Q, Huang Y, Lu Y, Peng Y, Zhang J, Feng G, Wang C, Liu L, and Dai Y. 2016. Tissue-specific and plasma microRNA profiles could be promising biomarkers of histological classification and TNM stage in non-small cell lung cancer. Thorac Cancer 7(3):348-354. 10.1111/1759-7714.12317

Reck M, Heigener DF, Mok T, Soria JC, and Rabe KF. 2013. Management of non-small-cell lung cancer: recent developments. Lancet 382(9893):709-719. 10.1016/s0140-6736(13)61502-0

Saito M, Schetter AJ, Mollerup S, Kohno T, Skaug V, Bowman ED, Mathé EA, Takenoshita S, Yokota J, Haugen A, and Harris CC. 2011. The association of microRNA expression with prognosis and progression in early-stage, non-small cell lung adenocarcinoma: a retrospective analysis of three cohorts. Clin Cancer Res 17(7):1875-1882. 10.1158/1078-0432.ccr-10-2961

Scott WJ, Howington J, Feigenberg S, Movsas B, and Pisters K. 2007. Treatment of non-small cell lung cancer stage I and stage II: ACCP evidence-based clinical practice guidelines (2nd edition). Chest 132(3 Suppl):234s-242s. 10.1378/chest.07-1378

Siriwardhana C, Khadka VS, Chen JJ, and Deng Y. 2019. Development of a miRNA-seq based prognostic signature in lung adenocarcinoma. Bmc Cancer 19(1):34. 10.1186/s12885-018-5206-8 
425

426

427

Stewart DJ. 2014. Wnt signaling pathway in non-small cell lung cancer. J Natl Cancer Inst 106(1):djt356. 10.1093/jnci/djt356

Sun S, Schiller JH, and Gazdar AF. 2007. Lung cancer in never smokers--a different disease. Nat Rev Cancer 7(10):778-790. 10.1038/nrc2190

Takamizawa J, Konishi H, Yanagisawa K, Tomida S, Osada H, Endoh H, Harano T, Yatabe Y, Nagino M, Nimura Y, Mitsudomi T, and Takahashi T. 2004. Reduced expression of the let-7 microRNAs in human lung cancers in association with shortened postoperative survival. Cancer Res 64(11):3753-3756. 10.1158/0008-5472.can-04-0637

Tibshirani R. 1997. The lasso method for variable selection in the Cox model. Stat Med 16(4):385-395. 10.1002/(sici)1097-0258(19970228)16:4<385::aid-sim380>3.0.co;2-3

Torre LA, Bray F, Siegel RL, Ferlay J, Lortet-Tieulent J, and Jemal A. 2015. Global cancer statistics, 2012. CA Cancer J Clin 65(2):87-108. 10.3322/caac.21262

Vansteenkiste J, Crinò L, Dooms C, Douillard JY, Faivre-Finn C, Lim E, Rocco G, Senan S, Van Schil P, Veronesi G, Stahel R, Peters S, and Felip E. 2014. 2nd ESMO Consensus Conference on Lung Cancer: early-stage non-small-cell lung cancer consensus on diagnosis, treatment and follow-up. Ann Oncol 25(8):1462-1474. 10.1093/annonc/mdu089

Wakelee HA, Chang ET, Gomez SL, Keegan TH, Feskanich D, Clarke CA, Holmberg L, Yong LC, Kolonel LN, Gould MK, and West DW. 2007. Lung cancer incidence in never smokers. J Clin Oncol 25(5):472-478. 10.1200/jco.2006.07.2983

Wang LL, and Zhang M. 2018. miR-582-5p is a potential prognostic marker in human non-small cell lung cancer and functions as a tumor suppressor by targeting MAP3K2. Eur Rev Med Pharmacol Sci 22(22):7760-7767. 10.26355/eurrev_201811_16397

Winter J, Jung S, Keller S, Gregory RI, and Diederichs S. 2009. Many roads to maturity: microRNA biogenesis pathways and their regulation. Nat Cell Biol 11(3):228-234. 10.1038/ncb0309-228

Wong N, and Wang X. 2015. miRDB: an online resource for microRNA target prediction and functional annotations. Nucleic Acids Res 43:D146-152. 10.1093/nar/gku1104

Xiong J, Guo S, Bing Z, Su Y, and Guo L. 2018. A Comprehensive RNA Expression Signature for Cervical Squamous Cell Carcinoma Prognosis. Front Genet 9:696. 10.3389/fgene.2018.00696

Xin X, Zhang Y, Ling F, Wang L, Sheng X, Qin L, and Zhao X. 2019. Identification of a nine-miRNA signature for the prognosis of Uveal Melanoma. Exp Eye Res 180:242-249. 
Xu RH, Wei W, Krawczyk M, Wang W, Luo H, Flagg K, Yi S, Shi W, Quan Q, Li K, Zheng L, Zhang H, Caughey BA, Zhao Q, Hou J, Zhang R, Xu Y, Cai H, Li G, Hou R, Zhong Z, Lin D, Fu X, Zhu J, Duan Y, Yu M, Ying B, Zhang W, Wang J, Zhang E, Zhang C, Li O, Guo R, Carter H, Zhu JK, Hao $X$, and Zhang K. 2017. Circulating tumour DNA methylation markers for diagnosis and prognosis of hepatocellular carcinoma. Nat Mater 16(11):1155-1161. 10.1038/nmat4997

Yang S, Liu Y, Li MY, Ng CSH, Yang SL, Wang S, Zou C, Dong Y, Du J, Long X, Liu LZ, Wan IYP, Mok T, Underwood MJ, and Chen GG. 2017. FOXP3 promotes tumor growth and metastasis by activating Wnt/ß-catenin signaling pathway and EMT in non-small cell lung cancer. Mol Cancer 16(1):124. 10.1186/s12943-017-0700-1

Yu G, Wang LG, Han Y, and He QY. 2012. clusterProfiler: an R package for comparing biological themes among gene clusters. Omics 16(5):284-287. 10.1089/omi.2011.0118

Yu T, Zhao Y, Hu Z, Li J, Chu D, Zhang J, Li Z, Chen B, Zhang X, Pan H, Li S, Lin H, Liu L, Yan M, He X, and Yao M. 2017. MetaLnc9 Facilitates Lung Cancer Metastasis via a PGK1-Activated AKT/mTOR Pathway. Cancer Res 77(21):5782-5794. 10.1158/0008-5472.can-17-0671

Zhang L, Liu Y, Chen XG, Zhang Y, Chen J, Hao ZY, Fan S, Zhang LG, Du HX, and Liang CZ. 2019. MicroRNA expression profile in chronic nonbacterial prostatitis revealed by next-generation small RNA sequencing. Asian J Androl 21(4):351-359. 10.4103/aja.aja_97_18

Zhang S, Yue W, Xie Y, Liu L, Li S, Dang W, Xin S, Yang L, Zhai X, Cao P, and Lu J. 2019. The four-microRNA signature identified by bioinformatics analysis predicts the prognosis of nasopharyngeal carcinoma patients. Oncol Rep 42:1767-1780. 10.3892/or.2019.7316

Zhao Y, Schetter AJ, Yang GB, Nguyen G, Mathé EA, Li P, Cai H, Yu L, Liu F, Hang D, Yang H, Wang XW, $\mathrm{Ke} \mathrm{Y}$, and Harris CC. 2013. microRNA and inflammatory gene expression as prognostic marker for overall survival in esophageal squamous cell carcinoma. Int J Cancer 132(12):2901-2909. 10.1002/ijc. 27954

Zheng D, Dai Y, Wang S, and Xing X. 2015. MicroRNA-299-3p promotes the sensibility of lung cancer to doxorubicin through directly targeting ABCE1. Int J Clin Exp Pathol 8(9):10072-10081.

Zheng R, Mao W, Du Z, Zhang J, Wang M, and Hu M. 2018. Three differential expression profiles of miRNAs as potential biomarkers for lung adenocarcinoma. Biochem Biophys Res Commun 507(14):377-382. 10.1016/j.bbrc.2018.11.046 
485 operating characteristic.

\section{Figure Legend:}

Zhu R, Lin W, Zhao W, Fan F, Tang L, and Hu Y. 2019. A 4-microRNA signature for survival prognosis in pediatric and adolescent acute myeloid leukemia. J Cell Biochem 120(3):3958-3968. 10.1002/jcb.27679

Figure 1. Construction of the six miRNAs predicted signature associated with OS of LUAD.

Distribution of 1,000 times resampled results in the top 8 OS-related miRNA (A); the Hazard ratio of enrolled OS-related miRNA (B) conducted by LASSO Cox regression analysis. OS, overall survival;

LASSO, the least absolute shrinkage and selection operator; LUAD, lung adenocarcinoma.

Figure 2. Evaluation of OS miRNA signature predictive performance. Kaplan-Meier curves of the low- and high-risk groups divided by six miRNA-based OS predictive signature in training cohort (A), and validation cohort (C); ROC curves of the low- and high-risk groups divided by six miRNA-based OS predictive in training cohort (B), and validation cohort (D).OS, overall survival; ROC, receiver

Figure 3. The comparison between the six-miRNA-based OS classifier and clinicopathological 
504

505

506

features. ROC curve showed the different performance of prognostic value between different variables in the overall set $(A)$, training set $(B)$, and validation set $(C)$. Nomogram was a synthesis model by combining the miRNA-based overall survival classifier and clinicopathological features. AUC, the area under the curve; ROC, receiver operating characteristic. OS, overall survival.

Figure 4. Stratified survival analyses of the LUAD patients according to the miRNA-based OS predicting classifier. Kaplan-Meier survival analyses for the patients with different characteristics including age, sex, tumor stage, and new tumor event after initial treatment (A-E) according to the miRNA-based OS predicting classifier. LUAD, lung adenocarcinoma; OS, overall survival.

Figure 5. Prediction of miRNAs down-stream target genes. We obtained the targeted genes of OS-related hsa-miR-4709, hsa-miR-582, hsa-miR-1468, hsa-miR-5571, hsa-miR-299 (A-E) from TargetScan, miRTarBase and miRDB, respectively, and overlapped the targeted genes to generated and visualized a new targeted gene cluster. OS, overall survival.

\section{Figure 6. Functional enrichment analysis depicted the biological pathways and processes} associated with OS correlated genes. Hallmark enrichment (A) and KEGG signaling pathways analysis (B) were conducted to classify the functions of these overlapped target genes of OS related hsa-miR-1468, hsa-miR-299, hsa-miR-4709, hsa-miR-5571, hsa-miR-582, respectively. OS, overall survival.

Fig. S1. Univariate Kaplan-Meier survival analyses for the overall survival-related miRNA candidates. 
523 Figure S2. Immunohistochemistry analysis for the CEP55 expression in non-cancerous tissue

524 adjacent to LUAD and LUAD tissue. A value of $P<0.05$ was regarded as statistically significant. ${ }^{* *}$

$525 P<0.01$. LUAD, lung adenocarcinoma.

526 Figure S3. Immunohistochemistry analysis for the MAP4K4 expression in non-cancerous

527 tissue adjacent to LUAD and LUAD tissue. A value of $P<0.05$ was regarded as statistically

528 significant. ${ }^{* \star *} P<0.001$. LUAD, lung adenocarcinoma.

529 Figure S4. Immunohistochemistry analysis for the RASA1 expression in non-cancerous tissue

530 adjacent to LUAD and LUAD tissue. A value of $P<0.05$ was regarded as statistically significant. ${ }^{* * *}$

$531 P<0.001$. LUAD, lung adenocarcinoma.

532 Figure S5. Immunohistochemistry analysis for the SOX9 expression in non-cancerous tissue

533 adjacent to LUAD and LUAD tissue. A value of $P<0.05$ was regarded as statistically significant. **

$534 P<0.01$. LUAD, lung adenocarcinoma.

535 Table S1. Raw material from TCGA.

536 Table S2. Risk score of each lung cancer patient.

537 Table S3. The means, variations, confidence intervals for the sensitivity, specificity and AUC 538 values. 
Figure 1

Construction of the six miRNAs predicted signature associated with OS of LUAD

Distribution of 1,000 times resampled results in the top 8 OS-related miRNA (A); the Hazard ratio of enrolled OS-related miRNA (B) conducted by LASSO Cox regression analysis. OS, overall survival; LASSO, the least absolute shrinkage and selection operator; LUAD, lung adenocarcinoma. 
A

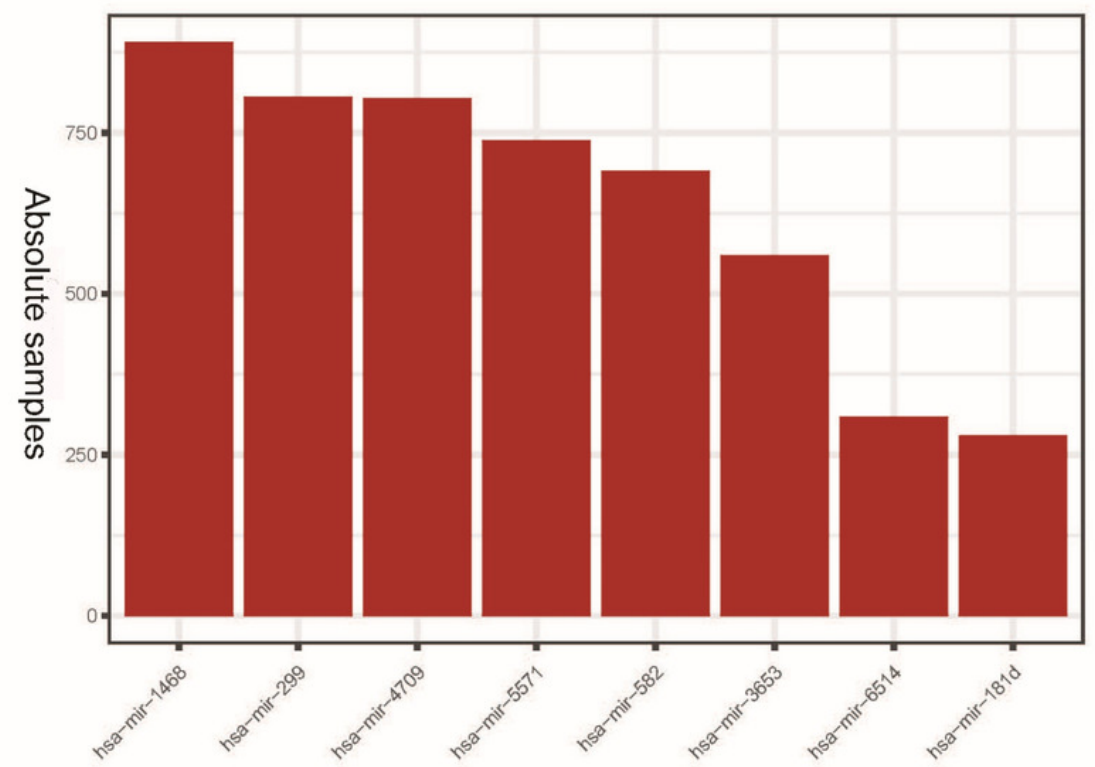

B

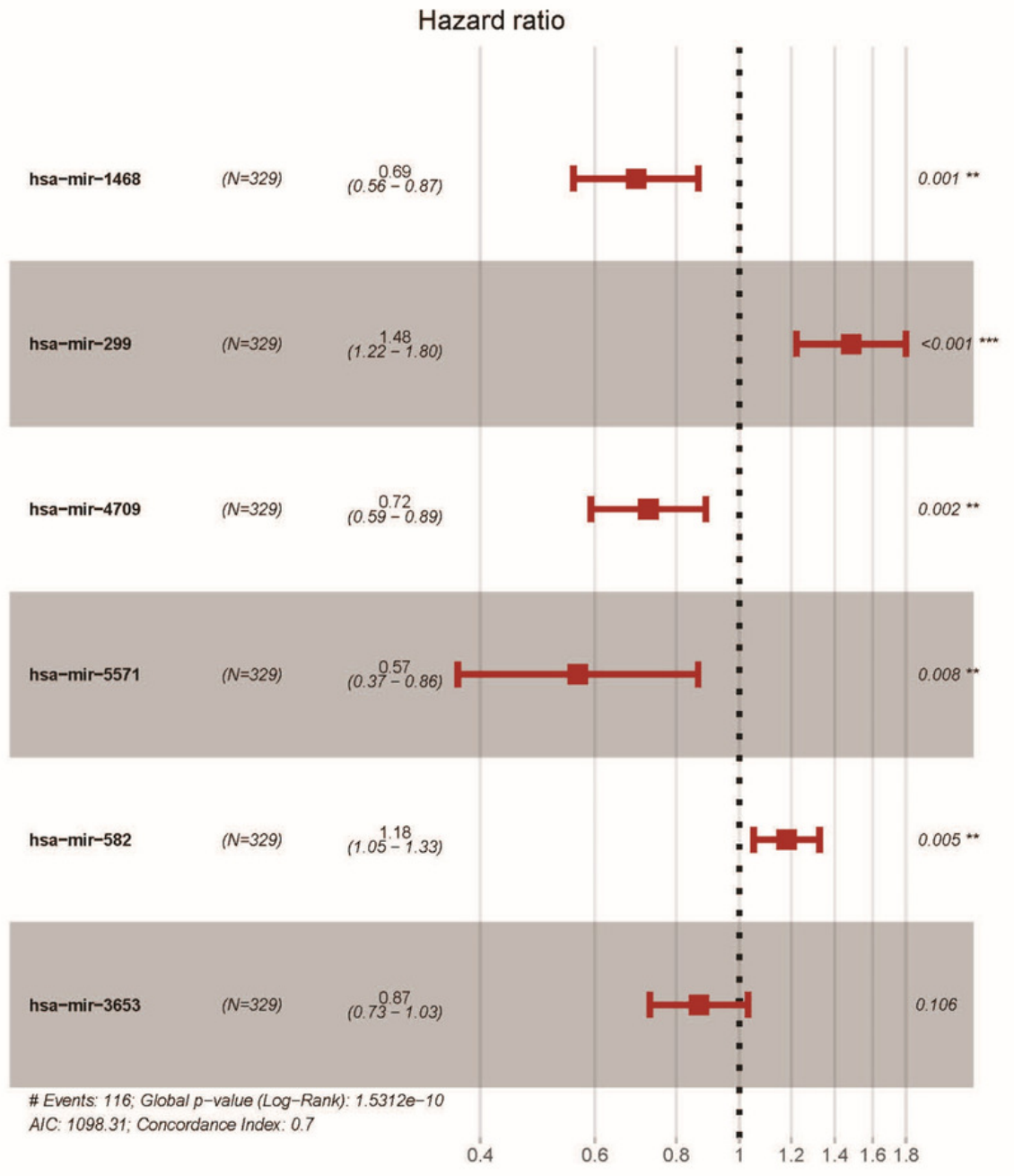


Figure 2

Evaluation of OS miRNA signature predictive performance

Kaplan-Meier curves of the low- and high-risk groups divided by six miRNA-based OS

predictive signature in training cohort $(\mathrm{A})$, and validation cohort $(\mathrm{C})$; ROC curves of the lowand high-risk groups divided by six miRNA-based OS predictive in training cohort (B), and validation cohort (D).OS, overall survival; ROC, receiver operating characteristic. 
A

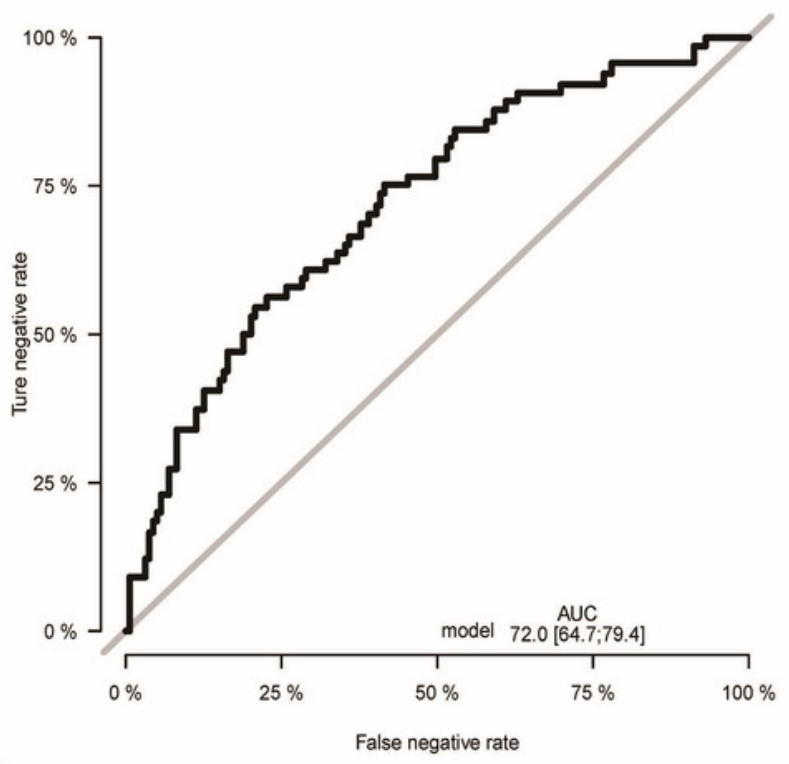

C

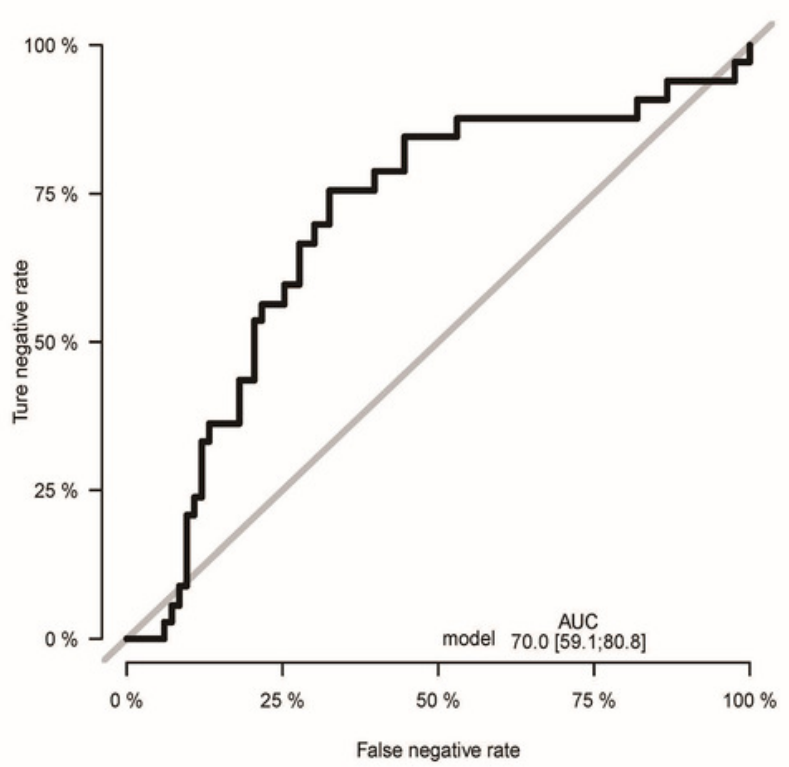

B

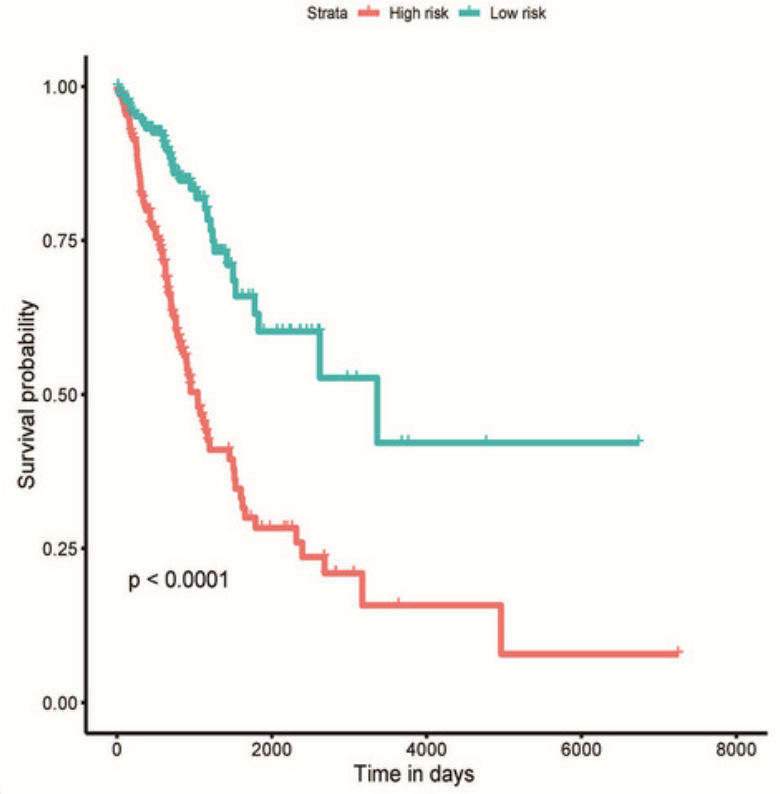

D

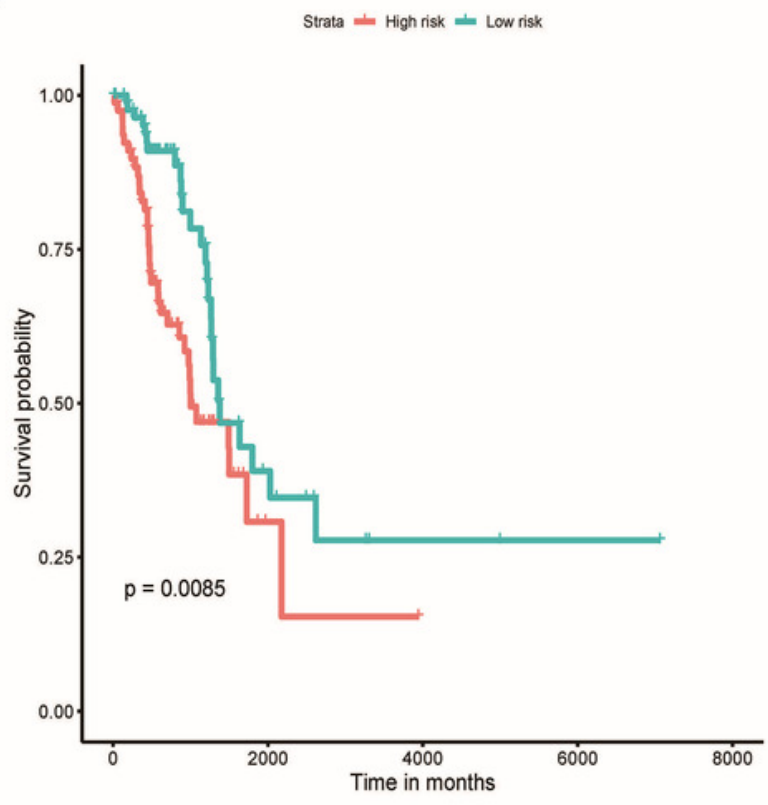


Figure 3

The comparison between the six-miRNA-based OS classifier and clinicopathological features.

ROC curve showed the different performance of prognostic value between different variables in the overall set (A), training set (B), and validation set (C). Nomogram was a synthesis model by combining the miRNA-based overall survival classifier and clinicopathological features. AUC, the area under the curve; ROC, receiver operating characteristic. OS, overall survival.

A

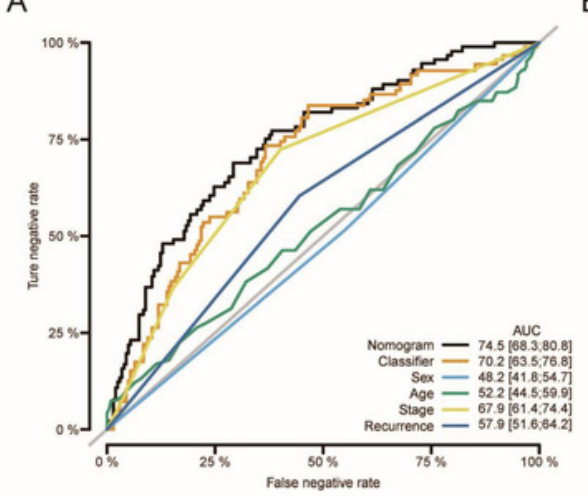

B

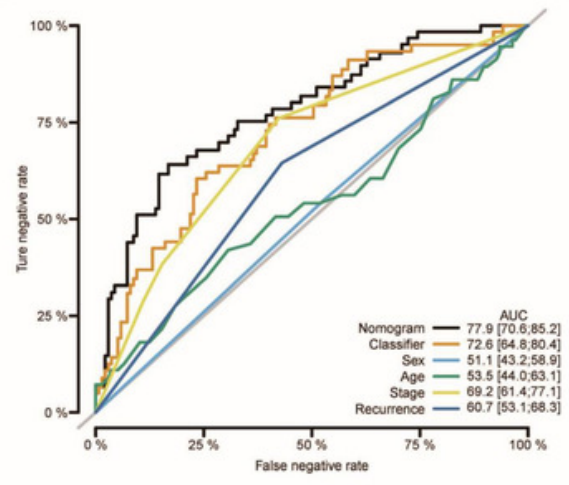

C

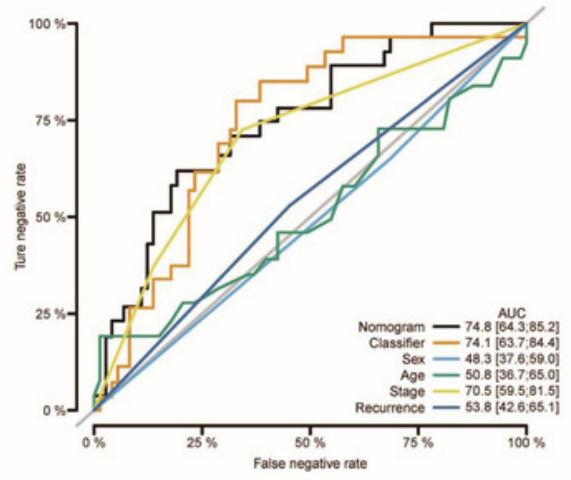


Figure 4

Stratified survival analyses of the LUAD patients according to the miRNA-based OS predicting classifier.

Kaplan-Meier survival analyses for the patients with different characteristics including age, sex, tumor stage, and new tumor event after initial treatment (A-E) according to the miRNAbased OS predicting classifier. LUAD, lung adenocarcinoma; OS, overall survival.
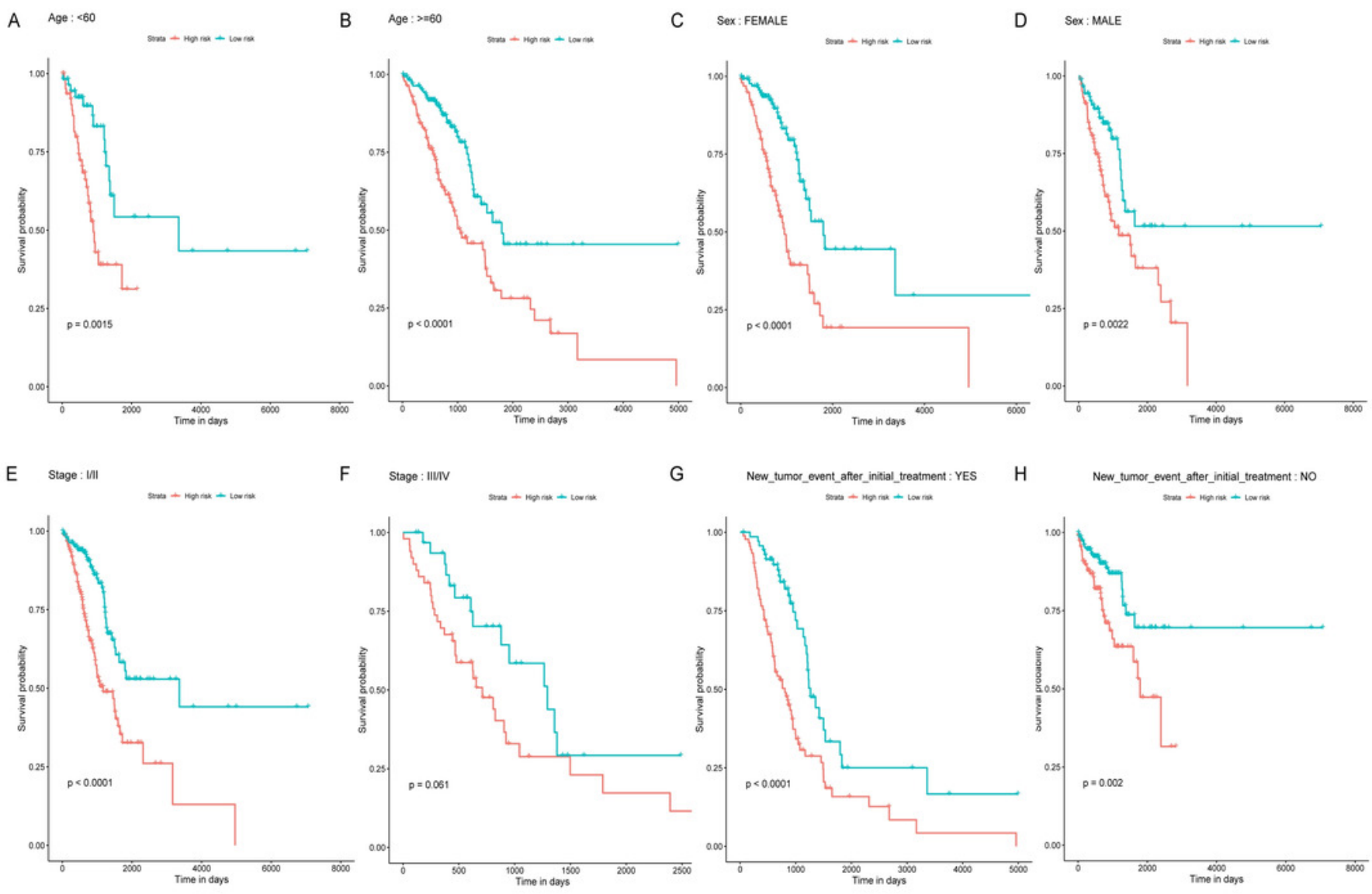


\section{Figure 5}

Prediction of miRNAs down-stream target genes

We obtained the targeted genes of OS-related hsa-miR-4709, hsa-miR-582, hsa-miR-1468, hsa-miR-5571, hsa-miR-299 (A-E) from TargetScan, miRTarBase and miRDB, respectively, and overlapped the targeted genes to generated and visualized a new targeted gene cluster. OS, overall survival 


\section{B hsa-mir-582}

A hsa-mir-4709

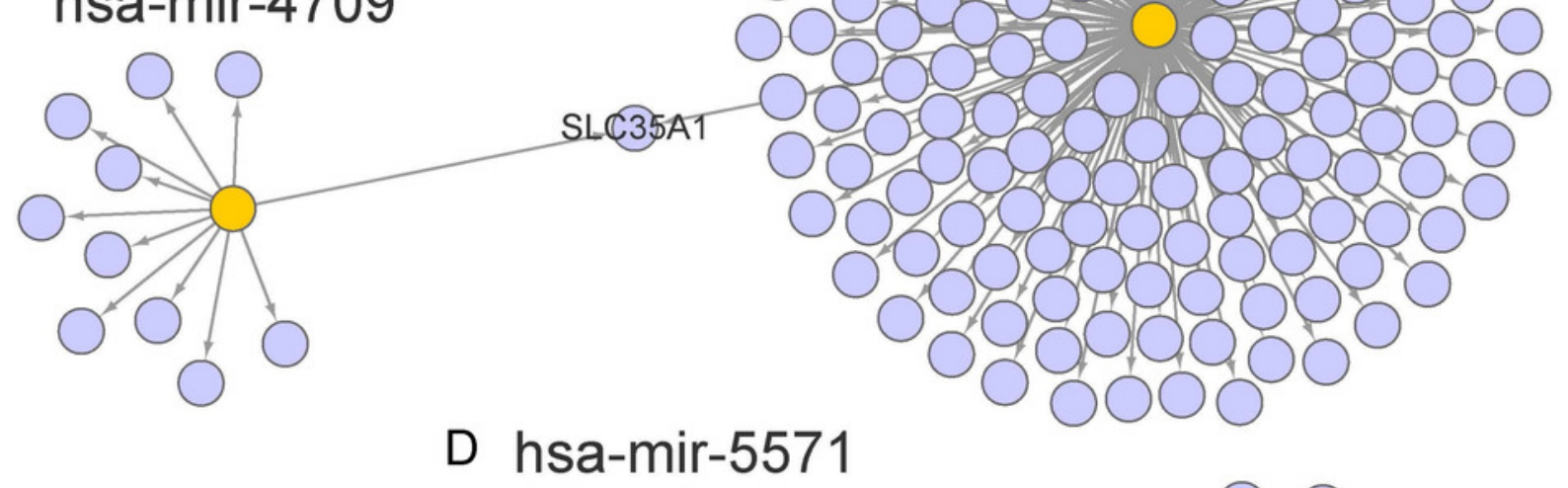

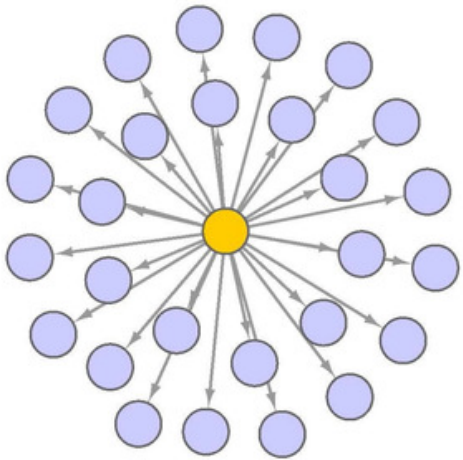

C hsa-mir-1468
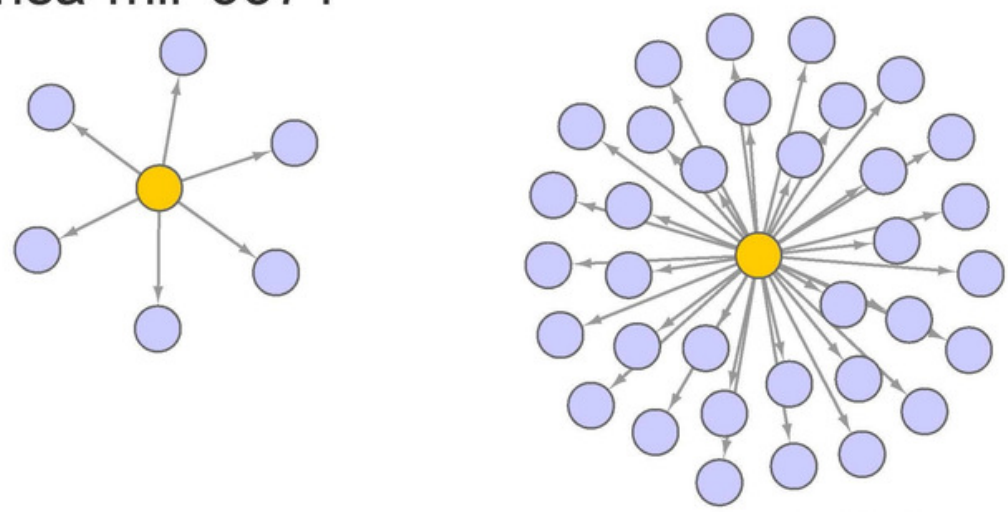

E hsa-mir-299 


\section{Figure 6}

Functional enrichment analysis depicted the biological pathways and processes associated with OS correlated genes

Hallmark enrichment (A) and KEGG signaling pathways analysis (B) were conducted to classify the functions of these overlapped target genes of OS related hsa-miR-1468, hsamiR-299, hsa-miR-4709, hsa-miR-5571, hsa-miR-582, respectively. OS, overall survival.

A

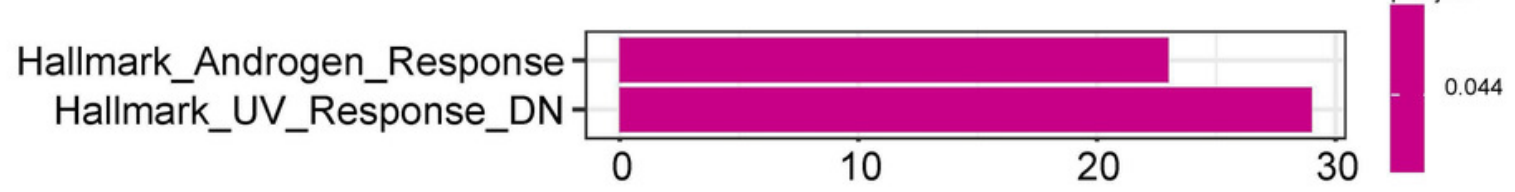

B

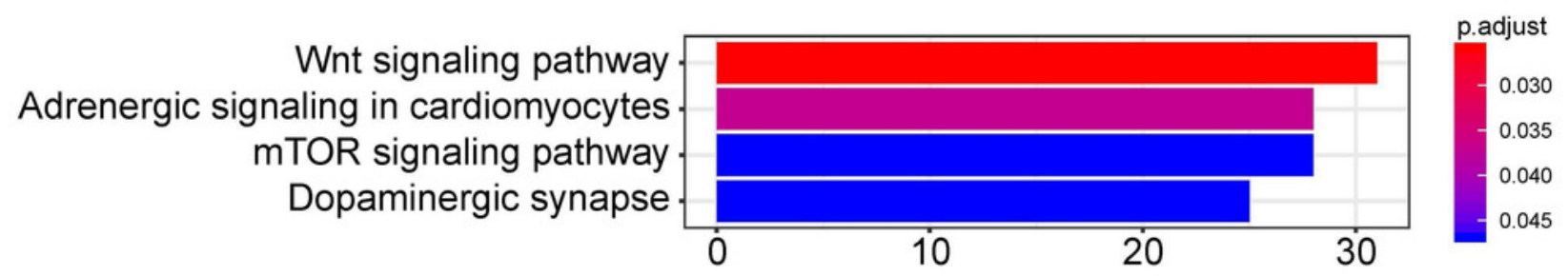

